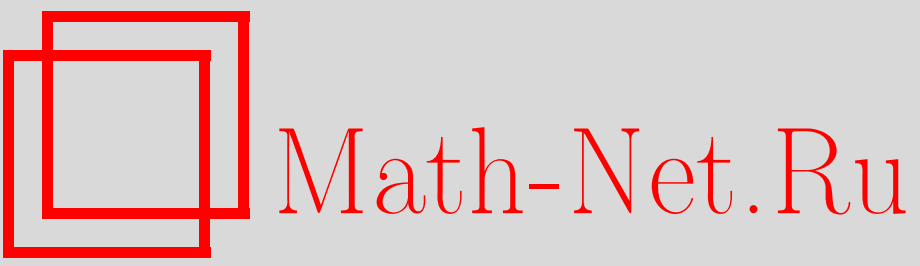

В. В. Корнев, А. П. Хромов, Абсолютная сходимость разложений по собственным и присоединенным функциям интегрального оператора с переменным пределом интегрирования, Изв. РАН. Сер. матем., 2005, том 69, выпуск 4, 59-74

DOI: https://doi.org/10.4213/im647

Использование Общероссийского математического портала Math-Net.Ru подразумевает, что вы прочитали и согласны с пользовательским соглашением

http://www.mathnet.ru/rus/agreement

Параметры загрузки:

IP : 107.22 .136 .117

26 апреля 2023 г., 13:55:09 


\author{
В.В. Корнев, А.П. Хромов
}

\title{
Абсолютная сходимость разложений по собственным и присоединенным функциям интегрального оператора с переменным пределом интегрирования
}

Для разложений по собственным и присоединенным функциям интегрального оператора $A f(x)=\int_{0}^{1-x} A(1-x, t) f(t) d t$ устанавливается аналог признака А. Зигмунда абсолютной сходимости тригонометрических рядов Фурье.

Библиография: 6 наименований.

1. Введение. Интегральньй оператор

$$
A f(x)=\int_{0}^{1-x} A(1-x, t) f(t) d t, \quad x \in[0,1]
$$

где $A(x, t)$ удовлетворяет условиям: $A(x, t) n$ раз непрерьвно дифференцируемо по $x$ и один раз по $t$, причем

$$
\left.\frac{\partial^{s}}{\partial x^{s}} A(x, t)\right|_{t=x}=\delta_{s, n-1}, \quad s=0, \ldots, n,
$$

$\delta_{s, n-1}$ - символ Кронекера, представляет собой простейший вид интегрального оператора, ядро которого имеет разрыв $(n-1)$-й производной по $x$ на линии $t=1-x$. Для такого оператора (и даже более общего, когда к (1) добавляется еше оператор $\left.\alpha \int_{0}^{x} A(x, t) f(t) d t\right)$ в работе [1] установлена равносходимость разложений по собственным и присоединенным функциям (с.п. ф.) и в обычный тригонометрический ряд Фурье. В [2] для него установлена базисность по Риссу в $L_{2}[0,1]$ системы с. п. ф. В настоящей статье устанавливается для разложений по с. п. ф. аналог известной теоремы А. Зигмунда [3, с. 614] об абсолютной сходимости тригонометрических рядов Фурье.

2. Резольвента оператора $A_{0}$. Обозначим через $A_{0}$ оператор

$$
A_{0} f(x)=\int_{0}^{1-x} \frac{(1-x-t)^{n-1}}{(n-1) !} f(t) d t
$$

Работа выполнена при финансовой поддержке гранта Президента РФ поддержки ведущих научных школ (проект НШ-1295.2003.1), РФФИ (проект № 03-01-00169) и программы “Университеты России" (проект УР.04.01.041). 
и через $R_{0, \lambda}=\left(E-\lambda A_{0}\right)^{-1} A_{0}$ его резольвенту Фредгольма ( $E$ - единичный оператор, $\lambda$ - спектральный параметр и для определенности $n=4 \nu+1)$.

Введем в рассмотрение следующую краевую задачу в пространстве векторфункций размерности два:

$$
\begin{gathered}
v^{(n)}(x)-\lambda \mathcal{D} v(x)=B F(x), \\
P v^{(j)}(0)+Q v^{(j)}(1)=0, \quad j=0, \ldots, n-1,
\end{gathered}
$$

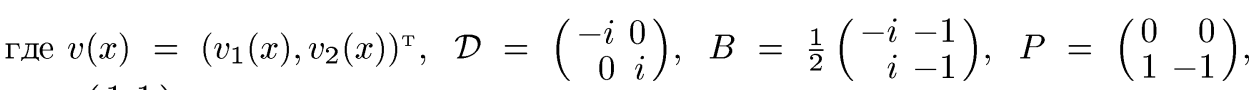
$Q=\left(\begin{array}{ll}1 & 1 \\ 0 & 0\end{array}\right), F(x)=\left(F_{1}(x), F_{2}(x)\right)^{\mathrm{T}}, F_{1}(x)=f(x), F_{2}(x)=f(1-x)$.

Лемма 1. Если $\lambda$ таково, что однородная задача, соответствующая задаче (2), (3), имеет только нулевое решение, то $R_{0, \lambda}$ существует и

$$
R_{0, \lambda} f(x)=v_{1}(x)+v_{2}(x) .
$$

ДоКАЗАТЕЛЬСТво. Краевая задача (2), (3) имеет и притом единственное решение. Положим $z_{1}(x)=v_{1}(x)+v_{2}(x)$ и $z_{2}(x)=i v_{1}(x)-i v_{2}(x)$. Тогда $z_{1}(x)$ и $z_{2}(x)$ удовлетворяют следующей краевой задаче:

$$
\begin{aligned}
& z_{1}^{(n)}(x)+\lambda z_{2}(x)=-f(1-x), \\
& z_{2}^{(n)}(x)-\lambda z_{1}(x)=f(x), \\
& z_{1}^{(j)}(1)=z_{2}^{(j)}(0)=0, \quad j=0, \ldots, n-1 .
\end{aligned}
$$

Заметим, что $z_{2}(1-x), z_{1}(1-x)$ также удовлетворяют (4). Поэтому в силу единственности решения системы $(4) z_{2}(x)=z_{1}(1-x)$ и для $z_{1}(x)$ получаем

$$
\begin{aligned}
& z_{1}^{(n)}(x)+\lambda z_{1}(1-x)=-f(1-x), \\
& z_{1}^{(j)}(1)=0, \quad j=0, \ldots, n-1 .
\end{aligned}
$$

Положим $\varphi(x)=-z_{1}(1-x)$. Тогда из (5) получаем

$$
\varphi^{(n)}(x)+\lambda z_{1}(x)=-f(x), \quad \varphi^{(j)}(0)=0, \quad j=0, \ldots, n-1 .
$$

Отсюда после $n$-кратного интегрирования следует, что

$$
\varphi(x)+\lambda \int_{0}^{x} \frac{(x-t)^{n-1}}{(n-1) !} z_{1}(t) d t=-\int_{0}^{x} \frac{(x-t)^{n-1}}{(n-1) !} f(t) d t .
$$

Значит,

$$
z_{1}(x)-\lambda \int_{0}^{1-x} \frac{(1-x-t)^{n-1}}{(n-1) !} z_{1}(t) d t=\int_{0}^{1-x} \frac{(1-x-t)^{n-1}}{(n-1) !} f(t) d t,
$$

T. e.

$$
z_{1}(x)-\lambda A_{0} z_{1}(x)=A_{0} f(x) .
$$

Отсюда в силу единственности решения уравнения (6) получаем, что $R_{0, \lambda}$ сушествует и $R_{0, \lambda} f(x)=z_{1}(x)$. Лемма доказана. 
Положим $\lambda=i \rho^{n}$, где $\rho$ принадлежит сектору $S=\left\{\rho \mid \arg \rho \in\left[0, \frac{2 \pi}{n}\right]\right\}$. Последний разобьем на четыре сектора $S_{j}=\left\{\rho \mid \arg \rho \in\left[\frac{\pi}{2 n}(j-1), \frac{\pi}{2 n} j\right]\right\}$, $j=1,2,3,4$. Для определенности будем рассматривать сектор $S_{1}$ (остальные рассматриваются аналогично). Обозначим через $\omega_{k}, k=1, \ldots, n$, корни $n$-й степени из 1 , занумерованные так, чтобы в $S_{1}$ выполнялись неравенства

$$
\operatorname{Re} \rho \omega_{1} \geqslant \operatorname{Re} \rho \omega_{2} \geqslant \cdots \geqslant \operatorname{Re} \rho \omega_{n_{0}} \geqslant 0>\operatorname{Re} \rho \omega_{n_{0}+1} \geqslant \cdots \geqslant \operatorname{Re} \rho \omega_{n} .
$$

Положим

$$
\begin{aligned}
g_{1}(x, t, \rho)= & \frac{1}{n \rho^{n-1}}\left\{\varepsilon(x, t) \sum_{k=n_{0}+1}^{n} \omega_{k} \exp \left(\rho \omega_{k}(x-t)\right)\right. \\
& \left.-\varepsilon(t, x) \sum_{k=1}^{n_{0}} \omega_{k} \exp \left(\rho \omega_{k}(x-t)\right)\right\}, \\
g_{2}(x, t, \rho)= & \frac{1}{n \rho^{n-1}}\left\{-\varepsilon(x, t) \sum_{k=1}^{n_{0}} \omega_{k} \exp \left(-\rho \omega_{k}(x-t)\right)\right. \\
& \left.+\varepsilon(t, x) \sum_{k=n_{0}+1}^{n} \omega_{k} \exp \left(-\rho \omega_{k}(x-t)\right)\right\},
\end{aligned}
$$

где $\varepsilon(x, t)=1$ при $t \leqslant x$ и $\varepsilon(x, t)=0$ при $t>x$.

Введем еще матрицы-функции:

$$
\begin{aligned}
& V_{k}(x, \rho)=\left(\begin{array}{cc}
\exp \left(\rho \omega_{k} x\right) & 0 \\
0 & \exp \left(-\rho \omega_{k} x\right)
\end{array}\right), \quad k=1, \ldots, n, \\
& g(x, t, \rho)=\left(\begin{array}{cc}
g_{1}(x, t, \rho) & 0 \\
0 & g_{2}(x, t, \rho)
\end{array}\right) .
\end{aligned}
$$

Обозначим

$$
U(\rho)=\left(U_{j k}(\rho)\right)_{1}^{n},
$$

где $U_{j k}(\rho)=U_{j}\left(V_{k}(x, \rho)\right), U_{j}(V)=P V^{(j-1)}(0)+Q V^{(j-1)}(1)$.

ТЕОРема 1. Если $\rho$ таково, что $U^{-1}(\rho)$ существует, то краевая задача (2), (3) имеет единственное решение, которое дается формулой

$$
\begin{aligned}
v(x, \rho)=- & \left(V_{1}(x, \rho), \ldots, V_{n}(x, \rho)\right) U^{-1}(\rho) \int_{0}^{1} U_{x}(g(x, t, \rho)) B F(t) d t \\
& +\int_{0}^{1} g(x, t, \rho) B F(t) d t,
\end{aligned}
$$

где $U_{x}(g)=\left(U_{1}^{\mathrm{T}}(g), \ldots, U_{n}^{\mathrm{T}}(g)\right)^{\mathrm{T}}$ (запись $U_{x}$ означает, что $U_{j}$ применяются к $g(x, t, \rho)$ по переменной $x)$. 
ДокАЗАТЕЛЬСТво. Матрицы-функции $V_{k}(x, \rho), k=1, \ldots, n$, образуют фундаментальную систему решений матричного уравнения

$$
V^{(n)}-\lambda \mathcal{D} V=0
$$

Непосредственная проверка показывает, что вектор-функция

$$
\int_{0}^{1} g(x, t, \rho) B F(t) d t
$$

является частным решением системы (2). Поэтому обшее решение этой системы имеет вид

$$
v(x, \rho)=\left(V_{1}(x, \rho), \ldots, V_{n}(x, \rho)\right) c+\int_{0}^{1} g(x, t, \rho) B F(t) d t
$$

где $c$ - произвольный постоянный вектор размерности $2 n$. После подстановки общего решения в краевые условия (3) для нахождения вектора $c$ получается система

$$
U(\rho) c+\int_{0}^{1} U_{x}(g(x, t, \rho)) B F(t) d t=0
$$

Находя отсюда $c$ и подставляя в (8), придем к формуле (7). Теорема доказана.

Обозначим $\Delta(\rho)=\operatorname{det} U(\rho)$ и через $\widetilde{\omega}_{k}, k=1, \ldots, 2 n$, числа $\omega_{k},-\omega_{k}, k=$ $1, \ldots, n$, занумерованные следуюшим образом:

$$
\operatorname{Re} \rho \widetilde{\omega}_{1} \geqslant \cdots \geqslant \operatorname{Re} \rho \widetilde{\omega}_{n} \geqslant 0 \geqslant \operatorname{Re} \rho \widetilde{\omega}_{n+1} \geqslant \cdots \geqslant \operatorname{Re} \rho \widetilde{\omega}_{2 n}
$$

Заметим, что $\widetilde{\omega}_{n}=\omega_{n_{0}}, \widetilde{\omega}_{n+1}=-\omega_{n_{0}}$.

ЛЕмма 2. Для определителя $\Delta(\rho)$ в области $S_{1}$ справедлива асимптотическая формула

$$
\Delta(\rho)=\rho^{n(n-1)}\left(a+b \exp \left(-2 \rho \widetilde{\omega}_{n}\right)+o(1)\right) \exp \left(\rho \sum_{k=1}^{n} \widetilde{\omega}_{k}\right)
$$

где а и $b$ - постоянные, отличные от нуля.

ДокаЗАТЕЛЬСТВо. Имеем

$$
U_{j k}(\rho)=\left(\begin{array}{cc}
\left(\rho \omega_{k}\right)^{j-1} \exp \left(\rho \omega_{k}\right) & \left(-\rho \omega_{k}\right)^{j-1} \exp \left(-\rho \omega_{k}\right) \\
\left(\rho \omega_{k}\right)^{j-1} & -\left(-\rho \omega_{k}\right)^{j-1}
\end{array}\right) .
$$


Отсюда видно, что после некоторой перестановки столбцов нечетные строки $\pm \Delta(\rho)$ будут иметь вид

$$
\left(\rho \widetilde{\omega}_{1}\right)^{j-1} \exp \left(\rho \widetilde{\omega}_{1}\right), \ldots,\left(\rho \widetilde{\omega}_{2 n}\right)^{j-1} \exp \left(\rho \widetilde{\omega}_{2 n}\right), \quad j=1, \ldots, n-1,
$$

а четные строки будут иметь вид

$$
\pm\left(\rho \widetilde{\omega}_{1}\right)^{j-1}, \ldots, \pm\left(\rho \widetilde{\omega}_{2 n}\right)^{j-1}, \quad j=1, \ldots, n-1,
$$

причем в каждом столбце каждая четная строка имеет один знак: плюс или минус. Разложим теперь $\pm \Delta(\rho)$ по теореме Лапласа по строкам с четными номерами. В итоге получим

$$
\Delta(\rho)=\rho^{n(n-1)} \sum_{j_{1}<\cdots<j_{n}} \gamma \exp \left(\rho\left(\widetilde{\omega}_{j_{1}}+\cdots+\widetilde{\omega}_{j_{n}}\right)\right)
$$

где $\gamma$ - постоянные коэффициенты (вообще говоря, различные). Отсюда, вынося экспоненты с неотрицательными действительными частями в показателях, получим формулу (9). Числа $a$ и $b$, как видно из структуры определителя $\Delta(\rho)$, равны с точностью до знака произведению определителей Вандермонда $n$-го порядка из наборов попарно различных чисел $\widetilde{\omega}_{j}$. Поэтому $a b \neq 0$. Лемма доказана.

Нули функции $a+b \exp \left(-2 \rho \widetilde{\omega}_{n}\right)$ обозначим через $\rho_{m}$. Тогда $\rho_{m}=a_{1}+b_{1} \pi m$, где $a_{1}=-\frac{1}{2 \widetilde{\omega}_{n}} \ln \left(-\frac{a}{b}\right), b_{1}=\frac{i}{\widetilde{\omega}_{n}}$. Очевидно, Re $b_{1}>0$. Удалим из $S_{1}$ нули $\rho_{m}$ вместе с круговыми окрестностями одного и того же достаточно малого радиуса $\delta$. Получившуюся область обозначим $S_{1, \delta}$.

ЛЕмма 3. В области $S_{1, \delta}$ при достаточно больиих $|\rho|$ справедлива оценка

$$
|\Delta(\rho)| \geqslant C|\rho|^{n(n-1)}\left|\exp \left(\sum_{k=1}^{n} \rho \widetilde{\omega}_{k}\right)\right|,
$$

где $C>0$ не зависит от $\rho$.

Это утверждение есть простое следствие леммы 2.

Обозначим $\sigma(x, \rho)=\exp \left(\rho \widetilde{\omega}_{k}(x-1)\right)$, если $k=1, \ldots, n$, и $\sigma(x, \rho)=\exp \left(\rho \widetilde{\omega}_{k} x\right)$, если $k=n+1, \ldots, 2 n$. Ясно, что $|\sigma(x, \rho)| \leqslant 1$.

ЛЕмма 4. В области $S_{1, \delta}$ при достаточно больиих $|\rho|$ для решения краевой задачи (2), (3) имеет место представление

$$
v(x, \rho)=\int_{0}^{1} G(x, t, \rho) B F(t) d t
$$

где $G(x, t, \rho)=g(x, t, \rho)+\frac{1}{\rho^{n-1}} H(x, t, \rho), \quad H(x, t, \rho)-$ матрица размера $2 \times 2$, компоненты которой являются линейными комбинациями всевозможных произведений $\sigma(x, \rho) \sigma(t, \rho)$ с коэффичиентами (не зависящими от $x$ u $t$ ), явяяюшимися ограниченными функииями $\rho$. 
ДокАЗАТЕЛЬСТво. По теореме 1 имеем

$$
\frac{1}{\rho^{n-1}} H(x, t, \rho)=-\left(V_{1}(x, \rho), \ldots, V_{n}(x, \rho)\right) U^{-1}(\rho) U_{x}(g(x, t, \rho))
$$

Рассмотрим $U_{x}(g(x, t, \rho))$. Имеем

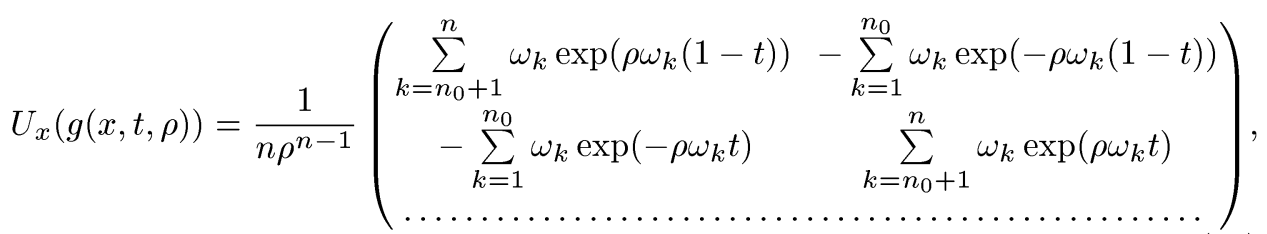

где многоточием обозначены $n-1$ клетки по две строки и два столбца. Каждая следуюшая отличается от предыдушей добавлением множителя $\rho$, а коэффищиенты $\omega_{k}$ первой клетки заменяются на $\pm \omega_{k}^{s}$ (т. е. на некоторые степени $\omega_{k}$ ). Поскольку все экспоненты, входящие в (11), есть $\sigma(t, \rho)$, то элементы первой клетки являются конечной суммой $\sigma(t, \rho)$ с постоянными коэффициентами, элементы второй - такой же суммой, умноженной на $\rho$, и так далее, элементы последней $n$-й клетки - указанными суммами, умноженными на $\rho^{n-1}$.

Рассмотрим теперь $J=\left(V_{1}(x, \rho), \ldots, V_{n}(x, \rho)\right) U^{-1}(\rho)$. Имеем

$$
U^{-1}(\rho)=\frac{1}{\Delta(\rho)}\left(\begin{array}{cccc}
A_{11} & A_{21} & \ldots & A_{2 n, 1} \\
\ldots \ldots \ldots \ldots \ldots \ldots \ldots \ldots \ldots \\
A_{1,2 n} & A_{2,2 n} & \ldots & A_{2 n, 2 n}
\end{array}\right)
$$

где $A_{j k}$ - алгебраическое дополнение элемента с индексом $(j, k)$ в матрице $U(\rho)$. Поэтому

$$
J=\frac{1}{\Delta(\rho)}\left(\begin{array}{ccc}
\sum_{j=1}^{n} A_{1,2 j-1} \exp \left(\rho \omega_{j} x\right) & \ldots & \sum_{j=1}^{n} A_{2 n, 2 j-1} \exp \left(\rho \omega_{j} x\right) \\
\sum_{j=1}^{n} A_{1,2 j} \exp \left(-\rho \omega_{j} x\right) & \ldots & \sum_{j=1}^{n} A_{2 n, 2 j} \exp \left(-\rho \omega_{j} x\right)
\end{array}\right)
$$

Из явного вида $\Delta(\rho)$ следует, что выражения

$$
\begin{array}{ll}
\frac{\rho^{1-s}}{\Delta(\rho)} A_{2 s-1,2 j-1} \exp \left(\rho \omega_{j} x\right), & \frac{\rho^{1-s}}{\Delta(\rho)} A_{2 s, 2 j-1} \exp \left(\rho \omega_{j} x\right), \\
\frac{\rho^{1-s}}{\Delta(\rho)} A_{2 s-1,2 j} \exp \left(-\rho \omega_{j} x\right), & \frac{\rho^{1-s}}{\Delta(\rho)} A_{2 s, 2 j} \exp \left(-\rho \omega_{j} x\right)
\end{array}
$$

равны $\sigma(x, \rho) O(1)$, где $O(1)$ не зависит от $x$. Из (10)-(12) получаем утверждение леммы. 
СЛЕДСТВИЕ 1. В области $S_{1, \delta}$ имеют место оценки

$$
\frac{\partial^{k}}{\partial x^{k}} G_{i j}(x, t, \rho)=O\left(\frac{1}{\rho^{n-1-k}}\right), \quad k=0, \ldots, n-1, \quad i, j=1,2
$$

əде $G_{i j}(x, t, \rho)$ - компоненты матрицы $G(x, t, \rho)$.

3. Резольвента оператора $A$. Введем в рассмотрение операторы

$$
T=\left(E+T_{1}\right)^{-1}-E, \quad T_{1} f(x)=\int_{0}^{x} \frac{\partial^{n}}{\partial x^{n}} A(x, t) f(t) d t .
$$

Из соотношения

$$
T_{1}(x, t)+T(x, t)+\int_{t}^{x} T(x, \tau) T_{1}(\tau, t) d \tau=0
$$

для ядер $T_{1}(x, t)$ и $T(x, t)$ операторов $T_{1}$ и $T$ следует, что $T(x, t)$ непрерывно дифференцируемо по $t$ и $T(x, x) \equiv 0$. Введем еще оператор $T_{t}^{\prime}$ :

$$
T_{t}^{\prime} f(x)=\int_{0}^{x} \frac{\partial}{\partial t} T(x, t) f(t) d t .
$$

Через $S_{\delta}$ обозначим область $S_{\delta}=\bigcup_{j=1}^{4} S_{j, \delta}$, где $S_{j, \delta}, j=2,3,4$, получаются аналогично $S_{1, \delta}$.

Лемма 5. В области $S_{\delta}$ ядро интегрального оператора $D^{n-1} S R_{0 \lambda} T_{t}^{\prime}$ есть o(1) при $|\rho| \rightarrow \infty$ равномерно относительно остальных переменных (здесь $\left.D=\frac{d}{d x}, S f(x)=f(1-x)\right)$.

Это утверждение следует из лемм 1,4 и [4, лемма 4].

Теорема 2. В области $S_{\delta}$ при больших $|\rho|$ справедлива формула

$$
R_{\lambda}=R_{0 \lambda}+R_{0 \lambda} T_{t}^{\prime}\left(E-D^{n-1} S R_{0 \lambda} T_{t}^{\prime}\right)^{-1} D^{n-1} S R_{0 \lambda}
$$

где $R_{\lambda}=(E-\lambda A)^{-1} A-$ резольвента Фредгольма оператора $A$.

ДокАЗАТЕЛЬСТво. Положим $y(x)=R_{\lambda} f(x)$. Тогда $(E-\lambda A) y=A f$. Отсюда имеем

$$
y(1-x)-\lambda \int_{0}^{x} A(x, t) y(t) d t=\int_{0}^{x} A(x, t) f(t) d t .
$$

Дифференцируя соотношение (13) $n$ раз, получим

$$
\begin{gathered}
D^{j} S y(x)-\lambda \int_{0}^{x} \frac{\partial^{j}}{\partial x^{j}} A(x, t) y(t) d t=\int_{0}^{x} \frac{\partial^{j}}{\partial x^{j}} A(x, t) f(t) d t, \quad j=0, \ldots, n-1, \\
D^{n} S y(x)-\lambda\left(E+T_{1}\right) y(x)=\left(E+T_{1}\right) f(x) .
\end{gathered}
$$


Применяя к обеим частям (15) оператор $(E+T)$, с учетом $(14)$ и равенства $T D^{n} S y=-T_{t}^{\prime} D^{n-1} S y$ получим

$$
\begin{gathered}
D^{n} S y-\lambda y=f(x)+T_{t}^{\prime} D^{n-1} S y, \\
y(1)=\cdots=y^{(n-1)}(1)=0 .
\end{gathered}
$$

Отсюда имеем

$$
y=R_{0 \lambda} f(x)+R_{0 \lambda} T_{t}^{\prime} D^{n-1} S y .
$$

Из соотношения (16) получаем

$$
D^{n-1} S y=D^{n-1} S R_{0 \lambda} f(x)+D^{n-1} S R_{0 \lambda} T_{t}^{\prime} D^{n-1} S y
$$

Отсюда по лемме 5 находим

$$
D^{n-1} S y=\left(E-D^{n-1} S R_{0 \lambda} T_{t}^{\prime}\right)^{-1} D^{n-1} S R_{0 \lambda} f(x) .
$$

Подставляя (17) в правую часть (16), получим утверждение теоремы.

4. Вспомогательные утверждения. Обозначим $\sigma(x, \rho, m)=\sigma\left(x, \rho+b_{1} m \pi\right)$, $w(x, t, \rho, m)=\varepsilon(x, t) \exp \left(\rho+b_{1} m \pi\right) \omega(x-t)$, если $\omega-$ одно из чисел $\widetilde{\omega}_{n+1}, \ldots, \widetilde{\omega}_{2 n} ;$ $w(x, t, \rho, m)=\varepsilon(t, x) \exp \left(\rho+b_{1} m \pi\right) \omega(x-t)$, если $\omega-$ одно из чисел $\widetilde{\omega}_{1}, \ldots, \widetilde{\omega}_{n}$, $\rho \in S_{1}$.

Введем следующие интегральные операторы:

$$
P_{m} g(x)=\int_{0}^{1} p(x, t, \rho, m) g(t) d t
$$

где $p(x, t, \rho, m)=\sigma(x, \rho, m) \sigma(t, \rho, m)$ или $p(x, t, \rho, m)=w(x, t, \rho, m)$.

Лемма 6 [5]. Пусть $\alpha \neq 0, \quad \operatorname{Re} \alpha \leqslant 0$. Тогда для любой функиии $f(x)$ из $L_{2}[0,1]$ выполнено неравенство

$$
\sum_{m=0}^{\infty}\left|a_{m}(f)\right|^{2} \leqslant c\|f\|_{2}^{2},
$$

где $a_{m}(f)=\int_{0}^{1} f(t) \exp (m \alpha t) d t,\|\cdot\|_{2}-$ норма в $L_{2}[0,1]$, постоянная $c>0$ зависит только от $\alpha$.

В работе [5] установлена лишь сходимость ряда слева в (18). Неравенство (18) следует из [6, с. 433].

ЛЕмма 7. Если $\rho$ принадлежит ограниченному множеству из $S_{1}$, то

$$
\sum_{m=0}^{\infty}\left|P_{m} g(x)\right|^{2} \leqslant c\|g\|_{2}^{2}
$$

где $c>0$ не зависит от $\rho$ и $x$. При этом ядра всех операторов $P_{m}$ в (19) отличаются лишь параметром т. 
ДокАЗАТЕЛЬСтво. Пусть сначала $p(x, t, \rho, m)=\sigma(x, \rho, m) \sigma(t, \rho, m)$. Тогда $P_{m} g(x)=\sigma(x, \rho, m) b_{m}(g, \rho)$, где $b_{m}(g, \rho)=\int_{0}^{1} g(t) \sigma(t, \rho, m) d t$. Поскольку $\sigma(t, \rho, m)=\sigma(t, \rho, 0) \sigma(t, 0, m)$, то

$$
b_{m}(g, \rho)=\int_{0}^{1} g_{1}(t, \rho) \sigma(t, 0, m) d t
$$

где $g_{1}(t, \rho)=g(t) \sigma(t, \rho, 0)$. Так как $|\sigma(t, \rho, 0)| \leqslant 1$, то $\left\|g_{1}\right\|_{2} \leqslant\|g\|_{2}$. Далее, $\sigma(t, 0, m)$ есть экспонента вида $\exp (z t m)$ или $\exp (z(1-t) m)$, где $z \neq 0$ и Re $z \leqslant 0$.

По лемме 6 имеем

$$
\sum_{m=0}^{\infty}\left|b_{m}(g, \rho)\right|^{2} \leqslant c\|g\|_{2}^{2}
$$

Поскольку $|\sigma(x, \rho, m)| \leqslant 1$, то $\left|P_{m} g(x)\right| \leqslant\left|b_{m}(g, \rho)\right|$ и утверждение леммы следует из (20).

Пусть теперь $p(x, t, \rho, m)=w(x, t, \rho, m)$ и для определенности $w(x, t, \rho, m)=$ $\varepsilon(t, x) \exp \left(\rho+b_{1} m \pi\right) \omega(x-t)$. Тогда

$$
P_{m} g(x)=\int_{0}^{1-x} g_{2}(x, t, \rho) \exp \left(-b_{1} m \pi t \omega\right) d t
$$

где $g_{2}(x, t, \rho)=g(t+x) \exp (-\rho \omega t)$. Фиксируем $x$. По лемме 6 имеем

$$
\sum_{m=0}^{\infty}\left|P_{m} g(x)\right|^{2} \leqslant c \int_{0}^{1-x}|g(t+x)|^{2} d t \leqslant c\|g\|_{2}^{2} .
$$

Лемма доказана.

Обозначим $M f=\int_{0}^{1} M(x, t) f(t) d t$, где ядро $M(x, t)$ есть продолженная нулем одна из функций $\frac{\partial}{\partial \eta} T(\xi, \eta)$, когда $\xi$ принимает значения $x$ или $1-x$, а $\eta$ принимает значения $t$ или $1-t$.

ЛЕмма 8. Если $\rho$ из ограниченного множества в $S_{1}$, то

$$
\sum_{m=0}^{\infty}\left|P_{m} M P_{m} g(x)\right| \leqslant c\|g\|_{2},
$$

где с >0 не зависит от $x$ и $\rho$. При этом ядра всех операторов $P_{m}$, стоящих перед $M$, отличаются лишь параметром $m$, таким же свойством обладают ядра всех операторов $P_{m}$, стоящих после $M$.

ДокАЗАТЕЛьСтво. Пусть сначала $p(x, t, \rho, m)=\sigma(x, \rho, m) \sigma(t, \rho, m)$ для $P_{m}$, стоящих после $M$ в $P_{m} M P_{m}$. Тогда $P_{m} g(x)=\sigma(x, \rho, m) b_{m}(g, \rho)$. Поэтому имеем

$$
M P_{m} g(x)=b_{m}(g, \rho) r_{m}(x)
$$


где $r_{m}(x)=\int_{0}^{1} M(x, t) \sigma(t, \rho, m) d t$. По лемме 6 получаем

$$
\sum_{m=0}^{\infty}\left|r_{m}(x)\right|^{2} \leqslant c
$$

где $c$ не зависит от $x$ и $\rho$. Далее по неравенству Коши-Буняковского в силу (20), (21) выполнено неравенство

поэтому

$$
\sum_{m=0}^{\infty}\left|r_{m}(x)\right|\left|b_{m}(g, \rho)\right| \leqslant c\|g\|_{2},
$$

$$
\begin{aligned}
\sum_{m=0}^{\infty}\left|P_{m} M P_{m} g(x)\right| & \leqslant \sum_{m=0}^{\infty} \int_{0}^{1}|p(x, t, \rho, m)|\left|M P_{m} g(t)\right| d t \\
& \leqslant \int_{0}^{1} \sum_{m=0}^{\infty}\left|M P_{m} g(t)\right| d t \leqslant c\|g\|_{2},
\end{aligned}
$$

и в этом случае лемма доказана.

Пусть теперь $p(x, t, \rho, m)=\sigma(x, \rho, m) \sigma(t, \rho, m)$ для $P_{m}$, стоящих перед $M$ в $P_{m} M P_{m}$. Тогда

$$
\begin{aligned}
\left|P_{m} M P_{m} g(x)\right| & \leqslant\left|\int_{0}^{1} \sigma(t, \rho, m) d t \int_{0}^{1} M(t, \tau) P_{m} g(\tau) d \tau\right| \\
& \leqslant \int_{0}^{1}\left|P_{m} g(\tau)\right|\left|\tilde{r}_{m}(\tau)\right| d \tau
\end{aligned}
$$

где $\tilde{r}_{m}(\tau)=\int_{0}^{1} M(t, \tau) \sigma(t, \rho, m) d t$. Отсюда по неравенству Коши-Буняковского и лемме 7 получим

$$
\begin{aligned}
\sum_{m=0}^{\infty}\left|P_{m} M P_{m} g(x)\right| & \leqslant \int_{0}^{1}\left(\sum_{m=0}^{\infty}\left|P_{m} g(\tau)\right|^{2}\right)^{1 / 2}\left(\sum_{m=0}^{\infty}\left|\tilde{r}_{m}^{2}(\tau)\right|\right)^{1 / 2} d \tau \\
& \leqslant c\|g\|_{2}\left(\int_{0}^{1} \sum_{m=0}^{\infty}\left|\tilde{r}_{m}^{2}(\tau)\right| d \tau\right)^{1 / 2}
\end{aligned}
$$

Однако по лемме 6 имеем

$$
\sum_{m=0}^{\infty}\left|\tilde{r}_{m}^{2}(\tau)\right| \leqslant c \int_{0}^{1}|M(t, \tau) \sigma(t, \rho, 0)|^{2} d t .
$$

Из неравенств $(22),(23)$ следует утверждение леммы в этом случае.

Пусть у двух операторов $P_{m}$ в $P_{m} M P_{m}$ ядро $p(x, t, \rho, m)=w(x, t, \rho, m)$, и для определенности пусть в обоих случаях

$$
w(x, t, \rho, m)=\varepsilon(x, t) \exp \left(\rho+b_{1} m \pi\right) \omega(x-t) .
$$

Тогда имеем

$$
P_{m} M P_{m} g(x)=\int_{0}^{1} P_{m} g(\tau) q_{m}(x, \tau) d \tau,
$$

где $q_{m}(x, \tau)=\int_{0}^{x} M(x-\xi, \tau) \exp \left(\rho+b_{1} m \pi\right) \omega \xi d \xi$. По лемме 6 получаем

$$
\sum_{m=0}^{\infty}\left|q_{m}(x, \tau)\right|^{2} \leqslant c
$$


где $c$ не зависит от $x, \tau, \rho$. Тогда по лемме 7

$$
\sum_{m=0}^{\infty}\left|P_{m} M P_{m} g(x)\right| \leqslant \int_{0}^{1} \sum_{m=0}^{\infty}\left|P_{m} g(\tau)\left\|q_{m}(x, \tau) \mid d \tau \leqslant c\right\| g \|_{2}\right.
$$

Лемма полностью доказана.

Лемма 9. Пусть $\rho \in S_{1, \delta}, \rho=\rho_{1}+b_{1} m \pi$ и $\rho_{1}$ принадлежит ограниченному множеству. Тогда

$$
\rho^{n-1-j} D^{j} R_{0 \lambda} f(x), \quad j=0, \ldots, n-1,
$$

есть конечная сумма с ограниченными по $\rho_{1}$ и $m$ коэффициентами операторов $P_{m} g$ при всевозможсных вариантах ядер $p\left(x, t, \rho_{1}, m\right)$ и $g(x)$ есть либо $f(x)$, либо $f(1-x)$.

ДокАЗАТЕЛЬСТво. По лемме 4 имеем

$$
\begin{aligned}
\rho^{n-1-j} D^{j} v(x, \rho)= & \rho^{n-1-j} \int_{0}^{1} \frac{\partial^{j}}{\partial x^{j}} g(x, t, \rho) B F(t) d t \\
& +\rho^{-j} \int_{0}^{1} \frac{\partial^{j}}{\partial x^{j}} H(x, t, \rho) B F(t) d t .
\end{aligned}
$$

Первое слагаемое в (24) есть вектор-функция, компоненты которой являются линейными комбинациями с постоянными коэффициентами функций $P_{m} g(x)$, когда $p\left(x, t, \rho_{1}, m\right)=w\left(x, t, \rho_{1}, m\right)$. Второе слагаемое в $(24)$ есть вектор-функция, компоненты которой являются линейными комбинациями функций $P_{m} g(x)$, когда

$$
p\left(x, t, \rho_{1}, m\right)=\sigma\left(x, \rho_{1}, m\right) \sigma\left(t, \rho_{1}, m\right)
$$

с коэффициентами, являющимися ограниченными функциями по $m$ и $\rho_{1}$. Утверждение леммы теперь следует из леммы 1.

5. Абсолютная равносходимость разложений по собственным функциям операторов $A$ и $A_{0}$. Обозначим через $\gamma_{m}$ окружности радиуса $\delta$ с центрами в $\rho_{m}$. Считаем, что такие $\rho_{m}$ и $\gamma_{m}$ найдены и в оставшихся секторах $S_{2}, S_{3}, S_{4}$ $\rho$-плоскости. Для прежних $\rho_{m}$ и вновь полученных сохраним прежние обозначения, т. е. все эти точки представим одной последовательностью $\left\{\rho_{m}\right\}$, занумерованной по правилу

$$
\left|\rho_{1}\right| \leqslant\left|\rho_{2}\right| \leqslant \ldots
$$

Несложно убедиться, что все $\sqrt[n]{-\lambda_{k} i}$ и $\sqrt[n]{-\lambda_{k}^{0} i}, \lambda_{k}, \lambda_{k}^{0}$ - характеристические числа операторов $A$ и $A_{0}$, достаточно большие по модулю, однократны и находятся по одному в каждом кружке $\gamma_{m}$. Обозначим через $\Gamma_{m}$ образы $\gamma_{m}$ в $\lambda$-плоскости. 
TEOPEMA 3. Существует натуральное $m_{0}$ такое, что для любой функции $f(x) \in L_{2}[0,1]$ ряд

$$
\sum_{m \geqslant m_{0}}\left|\int_{\Gamma_{m}}\left(R_{\lambda}-R_{0 \lambda}\right) f(x) d \lambda\right|
$$

сходится при всех $x \in[0,1]$ и его сумма ограничена.

ДокАЗАТЕЛьство. Ограничимся лишь случаем, когда $\gamma_{m} \in S_{1}$ (остальные случаи рассматриваются аналогично). Имеем

$$
\begin{aligned}
\sum_{m}\left|\int_{\Gamma_{m}}\left(R_{\lambda}-R_{0 \lambda}\right) f(x) d \lambda\right| & =\sum_{m}\left|\int_{\gamma_{m}}\left(R_{\lambda}-R_{0 \lambda}\right) f(x) n \rho^{n-1} d \rho\right| \\
& \leqslant n \sum_{m} \int_{\gamma_{m}}\left|\left(R_{\lambda}-R_{0 \lambda}\right) f(x)\right||\rho|^{n-1}|d \rho| \\
& =n \int_{\gamma_{0}} \Phi\left(f, \rho_{1}\right)\left|d \rho_{1}\right|
\end{aligned}
$$

где $\rho=\rho_{1}+b_{1} m \pi, \rho_{1} \in \gamma_{0}$,

$$
\Phi\left(f, \rho_{1}\right)=\sum_{m}\left|\left(R_{\lambda}-R_{0 \lambda}\right) f(x)\right|_{-\lambda i=\left(\rho_{1}+b_{1} m \pi\right)^{n}}\left|\rho_{1}+b_{1} m \pi\right|^{n-1} .
$$

Поскольку

$$
\left(E-D^{n-1} S R_{0 \lambda} T_{t}^{\prime}\right)^{-1}=E+\left(E-D^{n-1} S R_{0 \lambda} T_{t}^{\prime}\right)^{-1} D^{n-1} S R_{0 \lambda} T_{t}^{\prime},
$$

то по теореме 2

$$
R_{\lambda}-R_{0 \lambda}=R_{0 \lambda} T_{t}^{\prime} D^{n-1} S R_{0 \lambda} T_{t}^{\prime}+K D^{n-1} R_{0 \lambda} T_{t}^{\prime} D^{n-1} S R_{0 \lambda}
$$

где $K=R_{0 \lambda} T_{t}^{\prime}\left(E-D^{n-1} S R_{0 \lambda} T_{t}^{\prime}\right)^{-1} S$ (учли также, что $\left.D^{n-1} S=S D^{n-1}\right)$. По леммам $1,4,5 K$ есть интегральный оператор

$$
K f=\int_{0}^{1} K(x, t, \rho) f(t) d t
$$

с ядром $K(x, t, \rho)=O\left(\frac{1}{\rho^{n-1}}\right)$ в $S_{1, \delta}$. Из $(25)$ по лемме 9 можно оценить $\Phi\left(t, \rho_{1}\right)$ сверху конечной суммой рядов вида

$$
\sum_{m}\left|P_{m} M P_{m} g(x)\right|+\int_{0}^{1} \sum_{m}\left|P_{m} M P_{m} g(t)\right| d t
$$

где $P_{m}$ имеют тот же смысл, что и в лемме 8 . По лемме 8 эти ряды сходятся всюду и их суммы ограничены по $x$. Теорема доказана. 
6. Абсолютная сходимость разложений по с.п.ф. оператора $A$. Здесь мы установим аналог известной теоремы А. Зигмунда об абсолютной сходимости тригонометрических рядов Фурье для разложений по с. п. ф. интегрального оператора $A$.

Tеорема (А. Зигмунда [3, с. 614]). Пусть $f(x) \in V[0,1] \cap \operatorname{Lip} \alpha, f(0)=f(1)$ $u \alpha>0 ; a_{k}, b_{k}-$ коэффициенты ряда Фурье по тригонометрической системе $\{\cos (2 k \pi x)\} \cup\{\sin (2 k \pi x)\}$. Тогда

$$
\sum_{k=1}^{\infty}\left(\left|a_{k}\right|+\left|b_{k}\right|\right) \leqslant c \sqrt{L_{f}\left(V_{f}+M_{f}\right)}
$$

где с $>0$ зависит только от $\alpha, \quad V_{f}=\underset{0}{\mathrm{~V}}(f), \quad M_{f}=\max _{0 \leqslant x \leqslant 1}|f(x)|, \quad L_{f}-$ константа Липиица.

В работе [3] нет оценки (26), но она легко получается из рассуждений, приведенных в $[3$, с. $50-53,610,614]$.

ЛЕмма 10. Пусть $f(x)$ удовлетворяет условиям теоремы А. Зигмунда, a $g(x)$ непрерьвно дифференцируема. Тогда $F(x)=f(x) g(x) \in V[0,1] \cap \operatorname{Lip} \alpha$, причем $L_{F} \leqslant M_{1} L_{f}+M M_{2}, \quad V_{F} \leqslant V_{f} M_{1}+M M_{2}, \quad M=\|f(x)\|, \quad M_{1}=\|g(x)\|$, $M_{2}=\left\|g^{\prime}(x)\right\|,\|\cdot\|-$ норма в $C[0,1]$.

Эта лемма очевидна.

Лемма 11. Пусть $f(x) \in C[0,1],|f(x)| \leqslant c_{f} x^{\alpha}, \alpha>0$. Тогда

$$
\sum_{m=1}^{\infty}\left|\int_{0}^{1} f(t) \exp (\omega m t) d t\right| \leqslant c_{f} c(\alpha)
$$

əде $\omega=-\gamma+i \beta, \gamma>0, c(\alpha)$ зависит только от $\alpha$.

ДокаЗАТЕЛЬСТво. Имеем

$$
\left|\int_{0}^{1} f(t) \exp (\omega m t) d t\right| \leqslant c_{f} \int_{0}^{1} t^{\alpha} \exp (-\gamma m t) d t \leqslant \frac{c_{f}}{m^{1+\alpha}} \int_{0}^{\infty} t^{\alpha} \exp (-\gamma t) d t .
$$

Отсюда следует $(27), c(\alpha)=\int_{0}^{\infty} t^{\alpha} \exp (-\gamma t) d t \sum_{m=1}^{\infty} \frac{1}{m^{1+\alpha}}$.

Рассмотрим следующую задачу:

$$
\begin{aligned}
& u^{(n)}(x)-\lambda \mathcal{D} u(x)=B F(x), \\
& u^{(j)}(0)-u^{(j)}(1)=0, \quad j=0, \ldots, n-1,
\end{aligned}
$$

где $u(x)=\left(u_{1}(x), u_{2}(x)\right)^{\mathrm{T}}$. Роли $\rho_{m}$ для этой задачи выполняют точки $\tau_{m}=$ $2 m \pi b_{1}$, и мы их наряду с прежними $\rho_{m}$ удалим из $S_{1}$ вместе с круговыми окрестностями радиуса $\delta$ и получившуюся область по-прежнему обозначим $S_{1, \delta}$. Дальше рассуждаем, как в предыдущем пункте, т. е. получим контуры $\gamma_{m}$ и $\Gamma_{m}$, занумерованные в том же смысле.

Аналогично лемме 4 получается 
ЛЕМма 12. В области $S_{1, \delta}$ имеет место представление

$$
u(x)=u(x, \rho)=\int_{0}^{1} G_{1}(x, t, \rho) B F(t) d t
$$

əде $G_{1}(x, t, \rho)=g(x, t, \rho)+\frac{1}{\rho^{n-1}} H_{1}(x, t, \rho)$ и матрица $H_{1}(x, t, \rho)$ размера $2 \times 2$ имеет тот же вид, ито и $H(x, t, \rho)$ в лемме 4.

Лемма 13. Пусть $f(x) \in V[0,1] \cap \operatorname{Lip} \alpha$ u $f(0)=f(1)=0$. Тогда ряды $\sum_{m=1}^{\infty}\left\|P_{m}(x)\right\|$, где $\|\cdot\|$ - норма в $C[0,1]$, сходятся и их сум.мы ограничены по $\rho_{1}$, когда $\rho_{1}$ принадлежит ограниченному множеству. Ядра операторов $P_{m}$ в каждом ряде имеют вид $p\left(x, t, \rho_{1}, m\right)=\sigma\left(x, \rho_{1}, m\right) \sigma\left(t, \rho_{1}, m\right)$ и отличаются лишь параметром $\mathrm{m}$.

ДокаЗАТЕЛЬСТво. Имеем

$$
\left\|P_{m} f(x)\right\| \leqslant\left|\int_{0}^{1} \sigma\left(t, \rho_{1}, m\right) f(t) d t\right|
$$

Пусть $\sigma\left(t, \rho_{1}, m\right)=\sigma(t, \rho)=\exp \left(\rho \widetilde{\omega}_{k}(t-1)\right), \rho=\rho_{1}+b_{1} m \pi$ и $k=1, \ldots, n-1$. Тогда $\operatorname{Re} \rho \widetilde{\omega}_{k} \geqslant c|\rho|, c>0$. Отсюда $\operatorname{Re} b_{1} \pi \widetilde{\omega}_{k}>0$. Далее, имеем

$$
\int_{0}^{1} \sigma\left(t, \rho_{1}, m\right) f(t) d t=\int_{0}^{1} \psi\left(t, \rho_{1}\right) \exp \left(-b_{1} \pi m \widetilde{\omega}_{k} t\right) d t
$$

где $\psi\left(t, \rho_{1}\right)=f(1-t) \exp \left(-\rho_{1} \widetilde{\omega}_{k} t\right)$. Отсюда по лемме 11 получаем

$$
\sum_{m=1}^{\infty}\left|\int_{0}^{1} f(t) \sigma\left(t, \rho_{1}, m\right) d t\right| \leqslant c_{\psi} c(\alpha)
$$

Постоянная $c_{\psi}$ ограничена по $\rho_{1}$, и поэтому лемма в данном случае установлена.

Пусть теперь $k=n$. Тогда $b_{1} \widetilde{\omega}_{n}=i$, и поэтому

$$
\sum_{m}\left|\int_{0}^{1} \sigma\left(t, \rho_{1}, m\right) f(t) d t\right|=\sum_{m}\left|\int_{0}^{1} \psi\left(t, \rho_{1}\right) \exp (m \pi i t) d t\right|=\Sigma_{1}+\Sigma_{2}
$$

где $\Sigma_{1}$ - ряд по четным $m$, а $\Sigma_{2}$ - ряд по нечетным $m$. Рассмотрим $\Sigma_{1}$. По теореме Зигмунда $\Sigma_{1} \leqslant c \sqrt{L_{\psi}\left(V_{\psi}+M_{\psi}\right)}$. По лемме $10 L_{\psi}, V_{\psi}$ и $M_{\psi}$ ограничены по $\rho_{1}$. Рассмотрим $\Sigma_{2}$. Имеем

$$
\Sigma_{2}=\sum\left|\int_{0}^{1} \psi\left(t, \rho_{1}\right) \exp (\pi i t) \exp (2 m \pi i t) d t\right|
$$

и тем самым $\Sigma_{2}$ оценивается так же, как и $\Sigma_{1}$. Значит, и в этом случае лемма доказана. Случай $k \geqslant n+1$ исследуется аналогично. 
Лемма 14. Пусть $f(x)$ - такое жее, как в лемме 13. Существует номер $m_{0}$ такой, что ряд

$$
\sum_{m \geqslant m_{0}}\left|\int_{\Gamma_{m}}\left(R_{0 \lambda} f(x)-u_{1}(x, \rho)-u_{2}(x, \rho)\right) d \lambda\right|
$$

сходится всюду на $[0,1]$ и его сумма ограничена по $x$.

ДокАЗАТЕЛЬСТво. Пусть $\lambda=i \rho^{n}, \rho \in S_{1}$ и $\widetilde{\gamma}_{m}$ - часть контура $\gamma_{m}$, лежащая в $S_{1}$. Тогда существует $m_{0}$ такое, что по леммам 4 и 12 имеем

$$
\begin{aligned}
\sum_{m \geqslant m_{0}}\left|\int_{\widetilde{\gamma}_{m}}\left(R_{0 \lambda} f(x)-u_{1}(x, \rho)-u_{2}(x, \rho)\right) \rho^{n-1} d \rho\right| \\
=\sum_{m \geqslant m_{0}} \mid \int_{\widetilde{\gamma}_{m}}\left[\int_{0}^{1}(H(x, t, \rho) B F(t))_{1} d t+\int_{0}^{1}(H(x, t, \rho) B F(t))_{2} d t\right. \\
\left.\quad-\int_{0}^{1}\left(H_{1}(x, t, \rho) B F(t)\right)_{1} d t-\int_{0}^{1}\left(H_{1}(x, t, \rho) B F(t)\right)_{2} d t\right] d \rho \mid
\end{aligned}
$$

Поскольку $\sigma\left(1-t, \rho_{1}, m\right)=\sigma\left(t, \rho_{1}, m\right)$, то каждое слагаемое в правой части $(28)$ оценивается сверху конечной суммой следуюших интегралов от рядов:

$$
\int_{\widetilde{\gamma}_{0}} \sum_{m}\left\|P_{m} f(x)\right\|\left|d \rho_{1}\right|
$$

где операторы $P_{m}$ - те же, что и в лемме 13, и поэтому интегралы в $(29)$ конечны. Значит, правая часть (27) конечна и ограничена по $x$. Аналогично рассматриваются случаи, когда $\rho$ принадлежит остальным секторам $S_{2}, S_{3}, S_{4}$. Лемма доказана.

Лемма 15. Пусть $f(x)$ - такое жсе, как в лемме 13. Тогда ряд

$$
\sum_{m \geqslant m_{0}}\left|\int_{\Gamma_{m}}\left(u_{1}(x, \rho)+u_{2}(x, \rho)\right) d \lambda\right|
$$

сходится и его сумма ограничена по $x$.

ДокАЗАТЕЛЬСтво. Интеграл $\int_{\Gamma_{m}} u_{j}(x, \rho) d \lambda$ (если он не равен тождественному нулю) с точностью до постоянного множителя (не зависящего от $m$ ) есть отдельный член обычного тригонометрического ряда Фурье функции $(B F(x))_{j}$, и по теореме Зигмунда ряды $\sum\left|\int_{\Gamma_{m}} u_{j}(x, \rho) d \lambda\right|, j=1,2$, сходятся и их суммы ограничены по $x$. Лемма доказана.

Установим теперь основной результат настоящей статьи. 
Teоpema 4. Ecлu $f(x) \in V[0,1] \cap \operatorname{Lip} \alpha, \quad \alpha>0, u f(0)=f(1)=0$, mo ee ряд Фурье по с.п.ф. оператора А сходится абсолютно и сумма модулей его членов ограничена по $x$.

Утверждение теоремы вытекает из теоремы 3 и лемм 14 и 15 , если учесть, что с точностью до постоянного множителя ряд $\sum_{m \geqslant m_{0}} \int_{\Gamma_{m}} R_{\lambda} f(x) d \lambda$ может отличаться от ряда Фурье функции $f(x)$ по с. п. ф. оператора $A$ лишь на конечное число членов.

ЗАмечаниЕ. Условие $f(1)=0$ необходимо для сходимости разложений по с. п. ф. в точке $x=1$, а применение теоремы Зигмунда требует, чтобы $f(0)=f(1)$. Отсюда получается условие $f(0)=f(1)=0$.

\section{Список литературы}

1. Корнев В. В., Хромов А. П. О равносходимости разложений по собственным функциям интегральных операторов с ядрами, допускающими разрывы производных на диагоналях // Матем. сб. 2001. Т. 192. № 10. С. 33-50.

2. Курдюмов В. П., Хромов А. П. О базисах Рисса из собственных функций интегрального оператора с переменным пределом интегрирования // Докл. РАН. 2003. Т. 393. № 1. C. $14-17$.

3. Бари Н. К. Тригонометрические ряды. М.: Физматгиз, 1961.

4. Хромов А. П. Теоремы равносходимости для интегро-дифференциальных и интегральных операторов // Матем. сб. 1981. Т. 114 (156). №3. С. 378-405.

5. Кесельман Г. М. О безусловной сходимости разложений по собственным функциям некоторых дифференциальных операторов // Изв. вузов. Математика. 1964. № 2. С. 82-93.

6. Качмаж C., Штейнгауз $Г$. Теория ортогональных рядов. М.: Физматгиз, 1958.

Поступило в редакцию 05.03.2004 